\title{
Accuracy of the three-dimensional 3D Doppler over conventional 2D ultrasound in the diagnosis of morbid adherent placenta (MAP)
}

\author{
Original Hesham Mohamed Fathy, Ahmed Mohamed Bahaa, Ahmed Ali Alanwar, \\ Article \\ Ahmed Mohamed Salah Koura \\ Obstetrics and Gynecology Department, Faculty of Medicine, Ain Shams University
}

\begin{abstract}
Background: Two-dimensional ultrasound [2D] is in routine use in nearly most of the hospitals and many physician clinics as it offers a lot of benefits compared to other medical imaging techniques. Ultrasonography offers unique qualities including real-time imaging, physiologic measurement, use of non-ionizing radiation and no known bio-effects in the diagnostic range, while being non-invasive. Sonographic image quality has increasingly benefited from sophisticated computer technology.

Aim of the Work: Is to evaluate the diagnostic accuracy of three-dimensional (3D) power Doppler over conventional 2D Doppler in diagnosis of morbidly adherent placenta in patients with one or more previous cesarean section diagnosed as placenta previa.

Patients and Methods: The present study was carried out at Ain-Shams University Maternity Hospital. Women approached were recruited from Special Care Center of the fetus. After proper counseling, all women who were chosen for enrollment agreed to participate. The total number of pregnant women enrolled in the study was 120 women.

Results: In the following analysis, intraoperative and placental histopathology findings were considered as golden standard test when compared to the 2D and 3D uterine ultrasonography amongst the 120 studied cases.

Conclusion: The current study suggested that 3D power Doppler modality had a better screening capability for the prediction of the sequel of morbid placentation; namely, difficult placental delivery, considerable intraoperative blood loss, need for caesarean hysterectomy and bladder injury in women with placenta previa prior to cesarean (by its higher sensitivity and negative predictive value (NPV) and its lower false negative rates) when compared to the 2D ultrasound.
\end{abstract}

Key Words: Conventional 2D Ultrasound, Morbid Adherent Placenta, Three-Dimensional 3D Doppler.

Received: 23 August 2018, Accepted: 6 October 2018

Corresponding Author: Ahmed Mohamed Salah Koura, Obstetrics and Gynecology Department, Faculty of Medicine, Ain Shams University, E-mail: ahmedsalah_685@hotmail.com

ISSN: 2090-7265, November 2018, Vol.8, No. 4

\section{INTRODUCTION}

Two-dimensional ultrasound $[2 \mathrm{D}]$ is in routine use in nearly most of the hospitals and many physician clinics as it offers a lot of benefits compared to other medical imaging techniques. Ultrasonography offers unique advantages including real-time imaging, physiologic measurement, use of non-ionizing radiation, no known bio-effects in the diagnostic range while being non-invasive. Sonographic image quality has increasingly benefited from sophisticated computer technology (Schaap, 1999).

The challenging problem of abnormal placentation has become increasingly clinically significant in obstetric practice due to the dramatic rise in cesarean deliveries over the past four decades (Okada et al., 2009).

Morbidly-adherent placentas were manifested as placenta accreta, increta or percreta depending on the depth of placental invasion. These conditions presented high risk of severe obstetrical hemorrhage at delivery. The underlying pathology is due to defects in the decidua basalis caused by a variety of insults, such as previous surgery, excessive curettage or infection (Milosevic et al., 2009).

The incidence of morbidly adherent placentas increases as the frequency of cesarean sections increases. Imaging plays an important role in the antenatal detection of this co ndition

(Chou et al., 2009).

Placenta accreta occurs when placental trophoblasts invade the endometrium beyond the Nitabuch's layer of decidua basalis. Also, placenta increta occurs when placental trophoblasts invade the myometrium and placenta percreta occurs when placental trophoblasts invade the serosa (Abuhamad et al., 2014).

The three forms of morbidly adherent placenta (MAP); placenta accreta, increta and percreta, represent a significant 
obstetric challenge at times resulting in life-threatening bleeding and/or peripartum hysterectomy. The increasing rate of cesarean section (CS) deliveries correlates with the rising incidence of MAP (Daskalakis et al., 2007).

The risk increases in women with previous placenta previa and those with a previous CS. In addition to previous CS, a maternal age over 35 years, multiparity and previous curettage are risk factors associated with MAP (Mazouni et al., 2007).

This condition is often diagnosed during CS, upon placental removal, with unfavorable maternal outcome. The attempts to remove the placenta can cause severe uterine bleeding. An accurate prenatal diagnosis is required to reduce the risk of maternal/fetal morbidity and mortality (Tikkanen et al., 2011).

Successful management of this potentially catastrophic condition requires early antenatal diagnosis and referral to a tertiary institution where multidisciplinary expertise in perinatology, anesthesia, diagnostic radiology and blood transfusion services are available. Therefore, in such a condition, there is a need for reliable antenatal diagnoses. Also, when encountered unexpectedly at delivery, it will invariably lead to massive blood loss, multiple complications such as adult respiratory distress syndrome, Sheehan's syndrome, renal failure and death (Okada et al., 2009).

One area in which 3D ultra-sound seems to afford advantages over $2 \mathrm{D}$ ultrasound is in the imaging of abnormalities of the placenta, especially when the multislice capability of 3D ultrasound is combined with dynamic assessment of blood flow using power Doppler (Silver et al., 2006).

Despite the modern advances in imaging techniques, no single diagnostic technique affords complete assurance for the presence or absence of placenta accreta (ACOG, 2006). The diagnosis is most often made during the third stage of labor or on Cesarean delivery.

\section{AIM OF THE WORK}

The aim of this study is to evaluate the diagnostic accuracy of three-dimensional (3D) power Doppler over conventional 2D Doppler in diagnosis of morbidly adherent placenta in patients with one or more previous cesarean section diagnosed as placenta previa.

\section{PATIENTS AND METHODS}

The present study was carried out at Ain-Shams University Maternity Hospital. Women approached were recruited from Special Care Center of the fetus, Ain-Shams University Maternity Hospital.
After proper counseling, all women who were chosen for enrollment agreed to participate. The total number of pregnant women enrolled in the study was 120 women.

\section{Inclusion criteria:}

- $\quad$ Patients aged 2040- years (child bearing period).

- $\quad$ Pregnant women above 28 weeks gestation diagnosed as placenta previa by $2 \mathrm{D}$ ultrasound.

- Patients who have done one or more cesarean sections.

\section{Exclusion criteria:}

- Patients with bleeding disorders or on anticoagulant therapy.

- Systemic chronic diseases as chronic liver diseases, chronic kidney disease, hypertensive patients, diabetes.

- Mental illness.

\section{All patients underwent the following:}

History taking and general examination was done.

Decision making for the planned date of termination all were delivered by caesarean sections: (Either elective at term (3637- wks) or emergency in case of attack of antepartum hemorrhage).

\section{Perioperative preparations:}

- Preoperative CBC.

- Preoperative preparation of cross matched at least 4 units of packed RBCS.

- Counseling the patient and her family for the possibility of incidence of C.S hysterectomy, together with taking written high risk consent and consent for hysterectomy.

- At least 2 wide pore venous accesses were required.

- Senior obstetricians and anesthesiologists.

Statistical analysis: The collected data will be tabulated and statistically analyzed using SPSS program (Statistical Package for Social Sciences) software version 20.0. Descriptive statistics were done for numerical parametric data as mean $\pm \mathrm{SD}$ (standard deviation) and minimum and maximum of the range and for numerical non parametric data as median and 1 st and 3rd interquartile range, while they were done for categorical data 
as number and percentage. Inferential analyses were done for quantitative variables using independent t-test in cases of two independent groups with parametric data and Mann Whitney $U$ in cases of two independent groups with non parametric data. Receiver operating characteristic (ROC curve) analysis was used to find out the overall predictively of parameter and the best cut-off value with detection of sensitivity and specificity at this cut-off value. Inferential analyses were done for qualitative data using Chi square test for independent groups. The level of significance was taken at $\mathrm{P}$ value.

\section{RESULTS}

Table (1) shows demographic characteristics among the studied cases. 2D and 3D had significant moderate agreement with golden findings in prediction of morbidly adherent placenta; it was higher in $3 \mathrm{D}$ than in $2 \mathrm{D}$ as shown in table (6). Table (7) shows that 3D had higher diagnostic characteristics in prediction of morbidly adherent placenta. Table (9) shows that 3D had higher diagnostic characteristics in prediction of placenta percreta. 2D and 3D had significant moderate agreement with golden findings in prediction of hysterectomy; it was higher in 3D than in 2D. Table (11) and (fig. 1) show that 3D had higher diagnostic characteristics in prediction of hysterectomy.

Table 1: Demographic characteristics among the studied cases

\begin{tabular}{ccc}
\hline Variables & Mean \pm SD & Range \\
\hline Age (years) & $29.7 \pm 3.5$ & $20.0-39.0$ \\
BMI $\left(\mathrm{kg} / \mathrm{m}^{2}\right)$ & $26.8 \pm 1.4$ & $23.6-30.6$ \\
GA (weeks) & $35.3 \pm 2.4$ & $28.0-37.0$ \\
Parity & $2.4 \pm 0.7$ & $1.0-5.0$ \\
Previous CS & $1.5 \pm 0.6$ & $1.0-3.0$ \\
\hline
\end{tabular}

$\mathrm{N}=120$

Table 2: Agreement between 2Dand 3D Ultrasonography and golden findings in prediction of morbidly adherent placenta

\begin{tabular}{|c|c|c|c|c|c|}
\hline & \multirow[b]{2}{*}{ Test } & \multicolumn{2}{|c|}{ Golden } & \multicolumn{2}{|c|}{ Kappa } \\
\hline & & Positive & Negative & $\begin{array}{c}\text { Value } \\
(95 \% \mathrm{CI})\end{array}$ & $P$ \\
\hline \multirow{2}{*}{$2 \mathrm{D}$} & Positive & $29(24.2 \%)^{\mathrm{TP}}$ & $\begin{array}{c}18 \\
(15.0 \%)^{\mathrm{FP}}\end{array}$ & 0.560 & \multirow{2}{*}{$<0.001^{*}$} \\
\hline & Negative & $6(5.0 \%)^{\mathrm{FN}}$ & $\begin{array}{c}67 \\
(55.8 \%)^{\mathrm{TN}}\end{array}$ & $(0.408-0.712)$ & \\
\hline \multirow{2}{*}{$3 \mathrm{D}$} & Positive & $33(27.5 \%)^{\mathrm{TP}}$ & $\begin{array}{c}13 \\
(10.8 \%)^{\mathrm{FP}}\end{array}$ & 0.723 & \multirow{2}{*}{$<0.001^{*}$} \\
\hline & Negative & $2(1.7 \%)^{\mathrm{FN}}$ & $\begin{array}{c}72 \\
(60.0 \%)^{\mathrm{TN}}\end{array}$ & $(0.595-0.851)$ & \\
\hline
\end{tabular}

Percentages are from the total cases=120, TP: True positive, FP: False positive, FN: False negative, TN: True negative, CI: Confidence interval, *Significant
Table 3: Diagnostic characteristics of 2D and 3D ultrasonography in prediction of morbidly adherent placenta

\begin{tabular}{lcccc}
\hline \multirow{2}{*}{ Characters } & \multicolumn{2}{c}{$2 \mathrm{D}$} & \multicolumn{2}{c}{$3 \mathrm{D}$} \\
\cline { 2 - 5 } & Value & $95 \%$ CI & Value & \multicolumn{1}{c}{$95 \%$ CI } \\
\hline Sensitivity & $82.9 \%$ & $66.4 \%-93.4 \%$ & $94.3 \%$ & $80.8 \%-99.3 \%$ \\
Specificity & $78.8 \%$ & $68.6 \%-86.9 \%$ & $84.7 \%$ & $75.3 \%-91.6 \%$ \\
DA & $80.0 \%$ & $71.7 \%-86.7 \%$ & $87.5 \%$ & $80.2 \%-92.8 \%$ \\
Youden's index & $61.7 \%$ & $46.5 \%-76.9 \%$ & $79.0 \%$ & $68.1 \%-89.8 \%$ \\
PPV & $61.7 \%$ & $46.4 \%-75.5 \%$ & $71.7 \%$ & $56.5 \%-84.0 \%$ \\
NPV & $91.8 \%$ & $83.0 \%-96.9 \%$ & $97.3 \%$ & $90.6 \%-99.7 \%$ \\
LR+ & 3.91 & $2.53-6.06$ & 6.16 & $3.71-10.23$ \\
LR- & 0.22 & $0.10-0.45$ & 0.07 & $0.02-0.26$ \\
LR & 17.99 & $6.48-49.97$ & 91.38 & $19.50-428.26$ \\
\hline
\end{tabular}

CI: Confidence interval, YI: Youden's index, DA: Diagnostic accuracy, PPV: Positive Predictive value, NPV: Negative Predictive value, LR+: Positive likelihood ratio, LR-: Negative likelihood ratio, LR: Diagnostic odd ratio

Table 4: Diagnostic characteristics of 3D Ultrasonography in prediction of placenta percreta

\begin{tabular}{ccccc}
\hline \multirow{2}{*}{ Characters } & \multicolumn{2}{c}{$2 \mathrm{D}$} & \multicolumn{2}{c}{$3 \mathrm{D}$} \\
\cline { 2 - 5 } & Value & $95 \%$ CI & Value & $95 \%$ CI \\
\hline Sensitivity & $71.4 \%$ & $29.0 \%-96.3 \%$ & $100.0 \%$ & $59.0 \%-100.0 \%$ \\
Specificity & $77.0 \%$ & $68.1 \%-84.4 \%$ & $81.4 \%$ & $73.0 \%-88.1 \%$ \\
DA & $76.7 \%$ & $68.1 \%-83.9 \%$ & $82.5 \%$ & $74.5 \%-88.8 \%$ \\
Youden's index & $48.4 \%$ & $14.1 \%-82.8 \%$ & $81.4 \%$ & $74.2 \%-88.6 \%$ \\
PPV & $16.1 \%$ & $5.5 \%-33.7 \%$ & $25.0 \%$ & $10.7 \%-44.9 \%$ \\
NPV & $97.8 \%$ & $92.1 \%-99.7 \%$ & $100.0 \%$ & $96.1 \%-100.0 \%$ \\
LR+ & 3.10 & $1.74-5.53$ & 5.38 & $3.66-7.92$ \\
LR- & 0.37 & $0.11-1.20$ & 0.00 & $0.00-0.00$ \\
LR & 8.37 & $1.53-45.67$ & $>100.0$ & $>100.0->100.0$ \\
\hline
\end{tabular}

CI: Confidence interval, YI: Youden's index, DA: Diagnostic accuracy, PPV: Positive Predictive value, NPV: Negative Predictive value, LR+: Positive likelihood ratio, LR-: Negative likelihood ratio, LR: Diagnostic odd ratio

Table 5: Agreement between 2Dand 3D Ultrasonography and golden findings in prediction of hysterectomy

\begin{tabular}{cccccc}
\hline \multirow{2}{*}{ Test } & & \multicolumn{2}{c}{ Golden } & \multicolumn{2}{c}{ Kappa } \\
\cline { 2 - 6 } & & Positive & Negative & $\begin{array}{c}\text { Value } \\
(95 \% \mathrm{CI})\end{array}$ & $\mathrm{P}$ \\
\hline \multirow{3}{*}{ 2D } & Positive & $14(11.7 \%)^{\mathrm{TP}}$ & $\begin{array}{c}15 \\
(12.5 \%)^{\mathrm{FP}}\end{array}$ & 0.524 & \\
& & & 88 & $(0.337-0.710)$ & $<0.001 *$ \\
& Negative & $3(2.5 \%)^{\mathrm{FN}}$ & $(73.3 \%)^{\mathrm{TN}}$ & & \\
& & & 12 & & \\
$3 \mathrm{D}$ & Positive & $17(14.2 \%)^{\mathrm{TP}}$ & $(10.0 \%)^{\mathrm{FP}}$ & 0.682 & $<0.001^{*}$ \\
& & & 91 & $(0.521-0.844)$ & \\
\hline
\end{tabular}

Percentages are from the total cases $=120$, TP: True positive, FP: False positive, FN: False negative, TN: True negative, CI: Confidence interval, *Significant 
Table 6: Diagnostic characteristics of 3D Ultrasonography in prediction of hysterectomy

\begin{tabular}{ccccc}
\hline \multirow{2}{*}{ Characters } & \multicolumn{2}{c}{$2 \mathrm{D}$} & \multicolumn{2}{c}{$3 \mathrm{D}$} \\
\cline { 2 - 5 } & Value & $95 \%$ CI & Value & $95 \%$ CI \\
\hline Sensitivity & $82.4 \%$ & $56.6 \%-96.2 \%$ & $100.0 \%$ & $80.5 \%-100.0 \%$ \\
Specificity & $85.4 \%$ & $77.1 \%-91.6 \%$ & $88.3 \%$ & $80.5 \%-93.8 \%$ \\
DA & $85.0 \%$ & $77.3 \%-90.9 \%$ & $90.0 \%$ & $83.2 \%-94.7 \%$ \\
Youden's index & $67.8 \%$ & $48.4 \%-87.1 \%$ & $88.3 \%$ & $82.2 \%-94.5 \%$ \\
PPV & $48.3 \%$ & $29.4 \%-67.5 \%$ & $58.6 \%$ & $38.9 \%-76.5 \%$ \\
NPV & $96.7 \%$ & $90.7 \%-99.3 \%$ & $100.0 \%$ & $96.0 \%-100.0 \%$ \\
LR+ & 5.65 & $3.37-9.48$ & 8.58 & $5.04-14.61$ \\
LR- & 0.21 & $0.07-0.58$ & 0.00 & $0.00-0.00$ \\
LR & 27.38 & $7.01-106.87$ & $>100.0$ & $>100.0->100.0$ \\
\hline
\end{tabular}

CI: Confidence interval, YI: Youden's index, DA: Diagnostic accuracy, PPV: Positive Predictive value, NPV: Negative Predictive value, LR+: Positive likelihood ratio, LR-: Negative likelihood ratio, LR: Diagnostic odd ratio

\section{DISCUSSION}

Placenta previa increases the risk of antepartum, intrapartum and postpartum hemorrhage. For this reason, women with placenta previa are more likely to receive blood transfusions and undergo postpartum hysterectomy, uterine/iliac artery ligation or embolization of pelvic vessels to control bleeding. The risk is particularly high in those with previa-accreta (Lockwood and Russo-Stieglitz., 2015).

Rapid, significant loss of intravascular volume can lead to hemodynamic instability, decreased oxygen delivery, decreased tissue perfusion, cellular hypoxia, organ damage and death. The maternal mortality rate associated with placenta previa is less than 1 percent in resource-rich countries, but remains high in resource-poor countries where maternal anemia, lack of medical resources and home births are common (Lockwood and Russo-Stieglitz., 2015).

From a clinical stand point, placenta increta without bladder involvement is usually treated in the same way as placenta accreta, since the surgery and blood loss are usually the same and most obstetricians are able to manage these two clinical entities (Comstock et al., 2005).

In contrast, for patients with placenta percreta with tight bladder adherence or obvious bladder invasion, correct antenatal diagnosis and the appropriate management strategy can mean the difference between life and death. This is because infiltrating placental tissue invades the extensively vascularized lower uterine segment and the bladder, posing a serious risk of life-threatening hemorrhage (Comstock et al., 2005).
Prenatal diagnosis of placenta accretes/ percreta appears to improve outcome. In two retrospective series, women with pre-delivery diagnosis of accrete had significantly lower blood loss and transfusion requirements than women in whom the accreta was diagnosed during cesarean delivery (Warshak et al, 2010).

Prior Cesarean section (CS) and placenta previa (PP) are the two most important risk factors for placenta accrete. With the obviously increasing rates of CS, the incidence of both placenta previa (PP) and placenta accreta (PA) is steadily increasing in frequency concomitantly. Previous CS increases the odds of having a PA by about $8.7(\mathrm{Wu}$ et al., 2005). PP with previous CS compounds the risk. In women with known PP, $3 \%$ of those with no previous CS have PA compared to $11 \%$ of those with one previous CS. As the number of CS increases, so does the risk. Among women with PP, $40 \%$ of those with two CS and $61 \%$ of those with three previous CS have PA (Silver et al., 2006; Grobman et al., 2007).

Percentage of patients underwent cesarean hysterectomy was $14.2 \%$, By histopathology and intraoperative findings $13.3 \%$ was found to be placenta accreta, $10 \%$ was placenta increta and $5.8 \%$ was placenta accreta.

The current study showed that cases underwent cesarean hysterectomy had significantly high previous cesarean sections.

Previous CS was significantly different among placental findings; it was the highest in percreta, followed by increta then accreta.

Parity and no. of previous CS were significantly associated with higher incidence of difficult placental separation, intraoperative blood loss and CS hysterectomy. This is obviously reasonable, as with higher parity, there was a higher threshold for CS hysterectomy; and with increasing no. of previous CS, the possibility of having morbid placental invasion increased and this is in agreement with (Thia et al., 2007).

In spite of the lower cost and the higher feasibility of the 2D-GS ultrasound modality, its poor sensitivity led the practitioners to search for more advanced diagnostic modalities with relatively higher sensitivity and specificity.

Three-dimensional (3D) power Doppler (3DPD) ultrasound was studied as a diagnostic modality in antenatal prediction of placental adherence since the late 2000 and the early 2010 s.

The results of the current study showed a significant association between all criteria of the $3 \mathrm{D}$ power Doppler and presence of placental adherence, considerable 
intraoperative blood loss, need for added surgical steps, CS hysterectomy and bladder injury. All of the 3DPD ultrasound criteria tested in the current study showed a quite higher sensitivity and specificity in prediction of difficult placental separation when compared to $2 \mathrm{D}$ ultrasound findings (sensitivity $94.3 \%$, specificity $84.7 \%$, PPV 71.7\%, NPV between 97.3\%) and considerable intraoperative blood loss. When compared to 2D-GS ultrasound findings (sensitivity $82.9 \%$, specificity $78.8 \%$, PPV $61.7 \%$, NPV $91.8 \%$ ), the NPV represents the precision of diagnosis of placenta previa relating to the confidence with which clinicians can remove the placenta without concerns of severe bleeding.

The current study also showed a relatively high sensitivity and fair specificity of the 3DPD criteria in prediction of CS hysterectomy (sensitivity $100 \%$, specificity $88.3 \%$, PPV 58.6\%, NPV 100\%) and bladder invasion (sensitivity $100 \%$, specificity $81.4 \%$, PPV $25 \%$, NPV $100 \%$ ). (i.e. 3D Power Doppler has a good screening value).

\section{CONCLUSION}

In conclusion, the current study suggests that $3 \mathrm{D}$ power Doppler modality had a better screening capability for the prediction of the sequel of morbid placentation; namely, difficult placental delivery, considerable intraoperative blood loss, need for caesarean hysterectomy and bladder injury in women with placenta previa prior cesarean (by its higher sensitivity and negative predictive value (NPV); and its lower false negative rates when compared to the 2D ultrasound.

Despite the proper antenatal diagnosis of cases with morbid placentation, there is still significant incidence of complications as difficult placental separation, intraoperative blood loss, cesarean hysterectomy and urinary bladder injury.

\section{REFERENCES}

1. Abuhamad A (2014): Morbidly adherent placenta. Semin Perinatol; 37:359-64.

2. American College of Obstetricians and Gynecologists (ACOG) (2006): ACOG Practice Bulletin: Clinical Management Guidelines for ObstetricianGynecologists Number 76, October 2006: postpartum hemorrhage. Obstetrics and Gynecology; 108(4):1039.

3. Chou MM, Chen WC, Tseng JJ, Chen YF, Yeh TT, Ho ES (2009): Prenatal detection of bladder wall involvement in invasive placentation with sequential two-dimensional and adjunctive three-dimensional ultrasonography. Taiwanese Journal of Obstetrics and Gynecology; 48(1):38-45.
4. Chou MM, Ho ES, Lee YH (2000): Prenatal diagnosis of placenta previa accrete by transabdominal color Doppler ultrasound. Ultrasound Obstet Gynecol; $15: 28-35$

5. Comstock $\mathrm{CH}$, Love JJ Jr., Bronsteen RA, Lee W, Vettraino IM, Huang RR, Lorenz RP (2005): Sonographic detection of placenta accreta in the second and third trimesters of pregnancy. Am J Obstet Gynecol; 190: 1135-1140.

6. Daskalakis G, Anastasakis E, Papantoniou N, Mesogitis S, Theodora M, Antsaklis A (2007): Emergency obstetric hysterectomy. Acta Obstet Gynecol Scand; 86: 223-227.

7. Grobman WA, Gersnoviez R, Landon MB, et al (2007): Pregnancy outcomes for women with placenta previa in relation to the number of prior cesarean deliveries. Obstet Gynecol; 110:1249-1255.

8. Lockwood JC and Russo-Stieglitz K (2015): Clinical features, diagnosis, and course of placenta previa. In: Up ToDate, post, TW (Ed), Up ToDate, Waltham, MA.

9. Mazouni C, Gorincour G, Juhan V, Bretelle F (2007): Placenta accreta: a review of current advances in prenatal diagnosis. Placenta; 28: 599-603

10. Milosevic M, Zwahlen D, Jezioranski J, Chan P, Haider MA, Cho YB, Yeung I, Levin W, Manchul L, Fyles A (2009): Magnetic resonance imagingguided intracavitary brachytherapy for cancer of the cervix. International Journal of Radiation Oncology* Biology* Physics.; 74(4):1157-64.

11. Okada A, Morita Y, Fukunishi H, Takeichi K, Murakami $\mathrm{T}$ (2009): Non-invasive magnetic resonance-guided focused ultrasound treatment of uterine fibroids in a large Japanese population: impact of the learning curve on patient outcome. Ultrasound in Obstetrics and Gynecology; 34(5):579-83.

12. Resnik R (2015): Clinical features and diagnosis of the morbidly adherent placenta (placenta accreta, increta, and percreta) In: Up ToDate, Post, TW (Ed), Up ToDate, Waltham. MA.

13. Schaap AH, Bruinse HW, Smolders-de Haas H, Ertbruggen I, Treffers PE (1999): Vaginal delivery compared with caesarean section in early preterm breech delivery: a comparison of long term outcome. BJOG: An International Journal of Obstetrics and Gynaecology; 106(5):486-91. 
14. Silver RM, Landon MB, Rouse DJ, et al. (2006): Maternal morbidity associated with multiple repeat cesarean deliveries. Obstet Gynecol; 107:1226-1232.

15. Tikkanen M, Paavonen J, Loukovaara M, Stefanovic V (2011): Antenatal diagnosis of placenta accreta leads to reduced blood loss. Acta Obstet Gynecol Scand; 90: $1140-1146$.
16. Warshak CR, Ramos GA, Eskander R, Benirschke K, Saenz CC, Kelly TF, Moore TR and Resnik R (2010): Effect of predelivery diagnosis in 99 consecutive cases of placenta accreta. Obstet Gynecol; 115(1):65.

17. Wu S, Kocherginsky M, Hibbard JU (2005): Abnormal placentation: twenty-year analysis. Am J Obstet Gynecol 192:1458-1461. 